\title{
Characterization of DUT impedance in immunity test setups
}

\author{
Seyyed Ali Hassanpour Razavi and Stephan Frei \\ On-Board Systems Lab, TU Dortmund University, Dortmund, Germany \\ Correspondence to: S. A. Hassanpour Razavi (seyyed-ali.hassanpour-razavi@tu-dortmund.de) \\ Received: 22 January 2016 - Revised: 10 May 2016 - Accepted: 26 June 2016 - Published: 28 September 2016
}

\begin{abstract}
Several immunity test procedures for narrowband radiated electromagnetic energy are available for automotive components. The ISO 11452 series describes the most commonly used test methods. The absorber line shielded enclosure (ALSE) is often considered as the most reliable method. However, testing with the bulk current injection (BCI) can be done with less efforts and is often preferred. As the test setup in both procedures is quite similar, there were several trials for finding appropriate modifications to the $\mathrm{BCI}$ in order to increase the matching to the ALSE. However, the lack of knowledge regarding the impedance of the tested component, makes it impossible to find the equivalent current to be injected by the BCI and a good match cannot be achieved. In this paper, three approaches are proposed to estimate the termination impedance indirectly by using different current probes.
\end{abstract}

\section{Introduction}

In automotive electronic system configurations often the wire harness is assumed to be the main coupling path. This is taken into account in the EMC test setups described in the ISO 11452-2 (ALSE) and the ISO 11452-4 (BCI). In both methods, the electromagnetic energy is coupled to the wire harness. The ALSE method leads to a distributed electric and magnetic field coupling of the power along the entire length the wire harness. However, during BCI tests, the coupling appears only at one point. Moreover, the radiated immunity utilises the BCI for the lower frequency range from $1 \mathrm{MHz}$ to $400 \mathrm{MHz}$ and the ALSE for the higher frequency range from $80 \mathrm{MHz}$ to $18 \mathrm{GHz}$. In order to increase the performance of the $\mathrm{BCI}$, different investigations are published in Adams (1992) and Pignari (1996). In Grassi (2008), a double BCI method is proposed to reproduce the currents injected during the system level testing via an antenna by enforcing the equivalence between radiation and injection. Based on a theoretical investigation in Hassanpour (2014), it is shown that the current flowing into a device under test (DUT) during the ALSE can be reproduced with an acceptable accuracy by means of a $\mathrm{BCI}$ probe. This is done by injecting an appropriate power in to the $\mathrm{BCI}$ and an accurate positioning of the $\mathrm{BCI}$ probe along the wire harness, if the impedance of the DUT is available.

Apart from the coupling mechanism in the immunity test structures, the only variable part of the test setups in both ALSE and BCI remains the impedance of the terminating circuitry. The termination impedances may be obtained in a direct measurement, nevertheless, disconnecting the wire harness from the terminating circuit means additional preparation time and might cause problems to the proper system function. Based on a detailed analysis on the impedance measurement with current transformers in Junge (2009), applying a current transformer can be a convenient method for the indirect measurement of the DUT impedances in the immunity test setups. Undoubtedly, by obtaining this valuable information about a DUT with appropriate current probes and by knowing the coupled current during the antenna testing as a reference, the correlation between the ALSE and the BCI test methods can be improved. The problem of concern includes the estimation of the impedances terminating the wire harness ends with two different commercial current probes.

The number of wires involved in an immunity test setup is directly linked with the actual operation condition of the DUT. In case of a multi-wire transmission line, i.e. $n$ conductors over ground structure, the effects of the terminal network impedances on the left- and right side of test harness are contained in two $n \times n$ matrices. In general, these matrices are full, which means that there is cross coupling between all ports of the network. However, the most common case is the terminal network configuration where these impedance matrices are diagonal and the coupling occurs only along the 
multi-wire transmission line (Paul, 1994). Therefore, measuring the termination impedances for each single wire separately, provide the primary information to obtain the entire impedance matrix. Furthermore, it is assumed that the termination impedances consist of a passive RLC circuit. The above-mentioned issues are intended to assess the feasibility, strength and weakness of using current probes for measuring the impedance indirectly with current probes and serve as a primary research, which should be extended to the practically relevant case including a multi-wire harness and active circuit components.

In Sect. 2, three approaches for characterizing the termination impedances up to $500 \mathrm{MHz}$ for a single wire over ground structure are presented. In Sect. 3, the application of these methods is demonstrated for a measurement setup and the results are compared. More discussions and the conclusions are given in Sects. 4 and 5.

\section{Impedance measurement}

The test setup demanded in the ISO 11452, including a single wire and two unknown impedances $Z_{\mathrm{L}}$ and $Z_{\mathrm{R}}$ is illustrated in Fig. 1. The short conductor clamped by the BCI probe is considered as the secondary winding of the current transformer. In line with the idea of the indirect impedance measurement, the loop impedance can be considered as the entire impedance attached to the clamped conductor terminals. However, from another perspective, the loop impedance at any point along the wire can be considered as the accumulated transformed impedance of the termination networks. The transformed impedances along the wire to the measurement point are represented by $Z_{\mathrm{L}}{ }^{\prime}$ and $Z_{\mathrm{R}}{ }^{\prime}$. The first and the second methods presented in this section, determine the loop impedance as the primary value for the impedance measurement with different deembedding procedures. In contrast, the third method measures the impedance at each wire end separately based on current measurement.

\subsection{Single probe method (SPM)}

The most straightforward method is to apply a reflection measurement with a vector network analyzer (VNA) to determine the loop impedance. Measuring the input impedance with an impedance analyzer is an appropriate alternative for the reflection measurement with a VNA at this step, as the measured data by each instrument can be mathematically converted to each other. As depicted in Fig. 2 the BCI probe (FCC F-140) is connected to the VNA to measure the $\mathbf{S}$ parameter of the structure. The reflection parameter is corrected with the full 1-port calibration before starting the measurement. In order to remove the frequency response of the BCI probe, the measured one-port $\mathbf{S}$-parameter $s_{\text {meas }}$ is expanded to a two-port $\mathbf{S}$-parameter matrix $\mathbf{S}_{\mathrm{m}}$ for the measured frequencies with

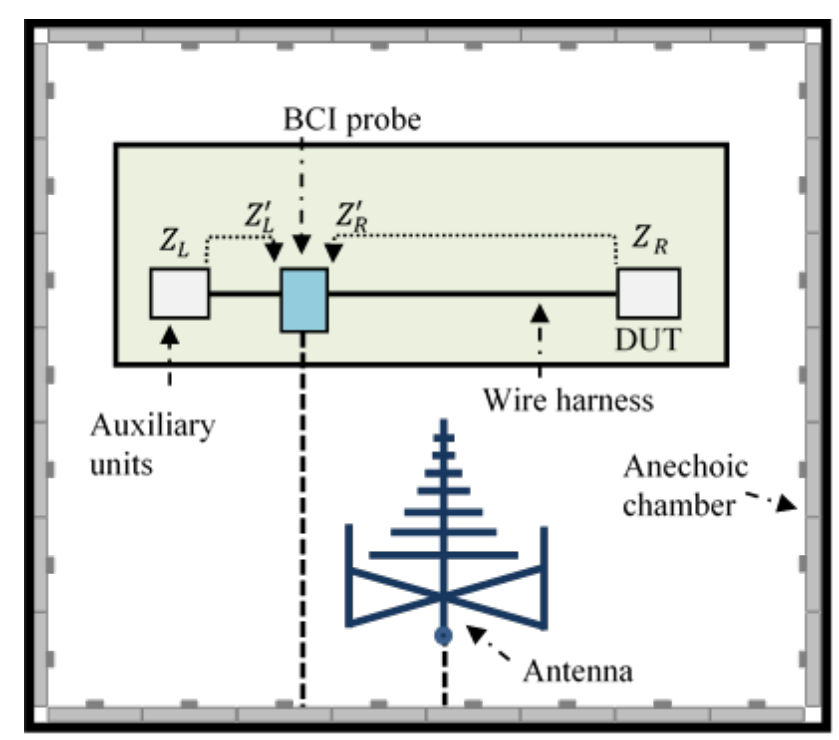

Figure 1. The proposed measurement setup for the ALSE and the BCI based on the ISO 11452 standard.

$\mathbf{S}_{\mathrm{m}}=\left(\begin{array}{cc}s_{\text {meas }} & 0 \\ 0 & -1\end{array}\right)$

The second port facilitates the mathematical operation on matrices and has no influence on the electrical characteristic of the actual network. The scattering matrix $\mathbf{S}_{\mathrm{m}}$ can now be converted to the $\mathbf{T}$-parameter matrix $\mathbf{T}_{\mathrm{m}}$ to simplify the deembedding procedure. The $\mathbf{T}$-parameter matrix can be split and expressed as

$\mathbf{T}_{\mathrm{m}}=\mathbf{T}_{\mathrm{BCI}} \mathbf{T}_{\text {loop }}$

where $\mathbf{T}_{\mathrm{BCI}}$ and $\mathbf{T}_{\text {loop }}$ denote the $\mathbf{T}$-parameter matrix of the BCI probe and the loop network respectively. The matrix $\mathbf{T}_{\mathrm{BCI}}$ can be obtained directly with the VNA in the calibration setup illustrated in Fig. 3 after a full 2-port calibration. With both matrices $\mathbf{T}_{\mathrm{m}}$ and $\mathbf{T}_{\mathrm{BCI}}$, the $\mathbf{T}$-parameter matrix of the loop network is determined as

$\mathbf{T}_{\text {loop }}=\mathbf{T}_{\mathrm{BCI}}^{-1} \mathbf{T}_{\mathrm{m}}$

As described before, the estimated loop impedance can be considered as the sum of both transformed impedances along the wire. Assuming a characterized termination on the left side and the BCI probe located as close as possible to the left termination, the extraction of the unknown impedance on the right side of the wire can be calculated using

$Z_{\mathrm{R}}=Z_{0} \frac{Z_{\text {loop }}-Z_{\mathrm{L}}-Z_{0} \tanh \left(\gamma l_{\mathrm{R}}\right)}{Z_{0}-\left(Z_{\text {loop }}-Z_{\mathrm{L}}\right) \tanh \left(\gamma l_{\mathrm{R}}\right)}$

where $Z_{0}$ and $\gamma$ indicate the characteristic impedance and the propagation constant of the wire over ground structure 


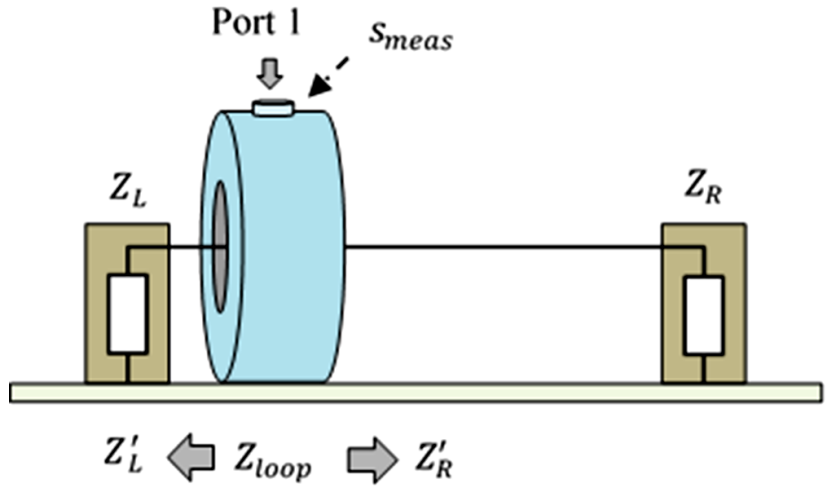

Figure 2. Measuring the reflection from the test setup with a VNA to obtain the loop impedance after deembedding the probe's frequency response.

respectively. The variable $l_{\mathrm{R}}$ is the distance between the $\mathrm{BCI}$ probe and the uncharacterized termination at the right side of the setup. In case of two unknown termination impedances, two measurements at different locations along the wire are required to estimate each impedance separately.

\subsection{Double probe method (DPM)}

In this method, two current probes (FCC F-140, FCC F65) are employed to characterize the loop impedance using two-port $\mathbf{S}$-parameter measurement with a VNA. The measurement structure is illustrated in Fig. 4. The frequency responses of both probes are deembedded using the calibration procedure described in Liu et al. (2003). The proposed calibration configuration is shown in Fig. 5. Two SMA terminations, short and $50 \Omega$, can be used in the calibration procedure to build the final equation calculating the loop impedance. In order to simplify the complex final equation, the interim value $S$ is defined as

$S=\frac{s_{21}}{1-s_{11}}$

where $s_{11}$ and $s_{21}$ indicate the corresponding entries of a twoport $\mathbf{S}$-parameter matrix. The loop impedance is then calculated using

$Z_{\text {loop }}=50 \Omega \frac{S_{50 \Omega}}{S_{\text {short }}-S_{50 \Omega}}\left(\frac{S_{\text {short }}}{S_{\mathrm{M}}}-1\right)$

where $S_{\text {short }}$ and $S_{50 \Omega}$ are the calculated values of $S$, if the calibration setup is terminated with the standard SMA terminations, short and $50 \Omega$, respectively. The value $S_{\mathrm{M}}$ is the calculated value of $S$ for the two-port $\mathbf{S}$-parameter measurement, if both probes are clamped around the wire in the actual test setup.

Apart from the calibration process, the estimation of impedances in this method does not differ from the SPM. In order to build up an equation system in case of two

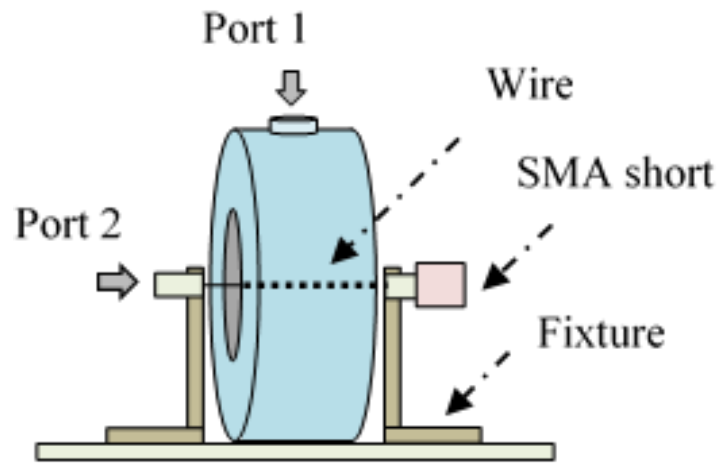

Figure 3. The proposed calibration setup to measure the probe's frequency response.

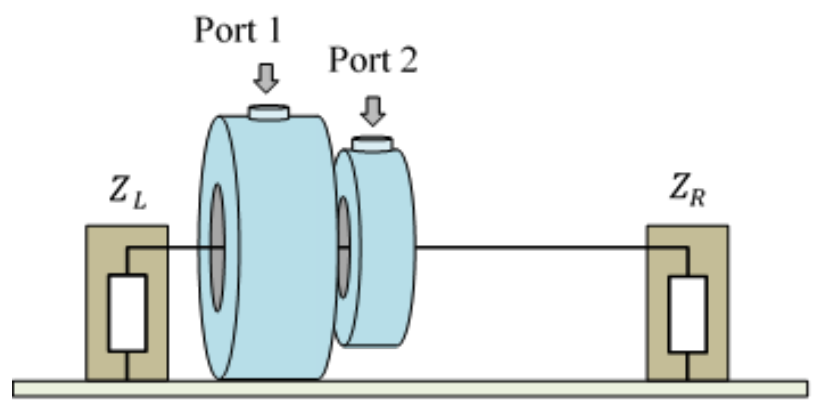

Figure 4. The measurement setup applying the DPM to calculate the loop impedance.

unknown impedances, the measurement structure including both probes should be placed at two different locations.

\subsection{Current distribution measurement (CDM)}

This method employs two current probes to measure the current distribution along the wire in order to extract the impedance of each termination directly. Therefore, the BCI probe (FCC F-140) is connected to the port 1 of the VNA to inject a certain interference level into the setup. The monitor current probe (FCC F-65) is connected to the port 2 to measure the transmission coefficient $s_{21}$ in the specified distances from the injection probe along the wire, as shown in Fig. 6. The number of measurements is directly linked with the measurement frequency range and the desired accuracy. In order to increase the accuracy for frequencies up to $500 \mathrm{MHz}$, the $\mathbf{S}$-parameter is measured with $3 \mathrm{~cm}$ step size. The measured transmission coefficient can be used to estimate the current magnitude $I$ at a certain position using

$I=s_{21} \frac{\sqrt{P_{\mathrm{Fwd}} \cdot 50 \Omega}}{Z_{\mathrm{T}}}$

where $P_{\text {Fwd }}$ is the injected input forward power of the VNA and $Z_{\mathrm{T}}$ is the transfer impedance of the monitor current probe. The ratio between the maximum and minimum mea- 


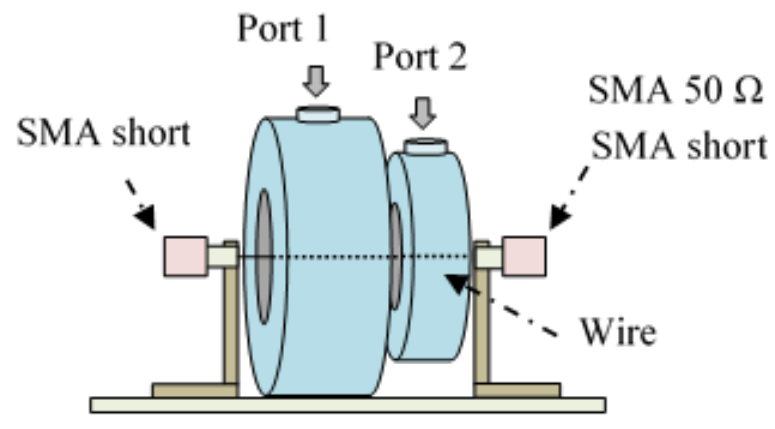

Figure 5. The calibration setup to deembed the frequency response of both probes from the measurement.

sured current magnitude $m$ can be expressed as

$m=\frac{I_{\max }}{I_{\min }}$

By knowing the characteristic impedance and the propagation constant of the wire, the termination impedance $Z$ can be calculated as

$Z=Z_{0} \cdot \frac{\frac{1}{m}-\tanh \left(\gamma l_{1 \min }\right)}{1-\frac{1}{m} \tanh \left(\gamma l_{1 \min }\right)}$,

where $l_{1 \text { min }}$ is the distance of the first minimum current magnitude from the end of wire. This method requires a wire length of at least $\lambda / 4$. In order to cover the lower frequencies, the length of the wire should be increased. The accuracy of the estimated results is extremely sensitive to the current distribution resolution. The main advantage of this method is that the impedance for each termination can be extracted directly. Additionally, the measurement can also be performed directly by using a signal generator and a spectrum analyzer instead of a VNA, to measure the current magnitude along the wire.

\section{Application and experimental results}

In Sect. 2, three different methods for an estimation of the termination impedances are presented. To investigate the reliability of each method, a setup based on ISO 11452 standard with a single wire attached between two grounded metal fixtures is prepared. The setup is terminated with a $50 \Omega$ SMA resistor at the left side. The right side of the setup is terminated with two small PCBs including a $10 \Omega$ and a $470 \Omega$ SMD resistors successively, to perform two separate measurements. The characteristic impedance and the propagation constant of the single wire over ground is characterized in the frequency range from 1 to $500 \mathrm{MHz}$ based on a twoport $\mathbf{S}$-parameter measurement. The value of the termination impedance at the right side is assumed to be unknown. For the SPM and DPM, the current probes are located at the left

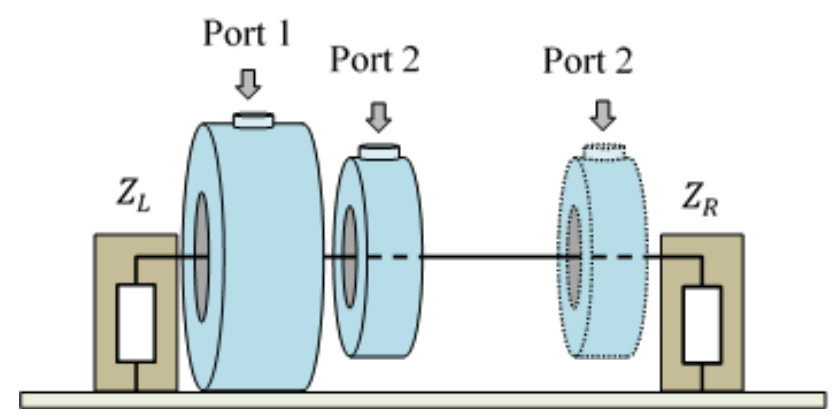

Figure 6. Appling the CDM along a wire to estimate the impedance of terminations.

side of the setup close to the left fixture. All of three methods are performed with a constant forward power of $10 \mathrm{dBm}$. The impedance of each PCB is measured directly in a separate measurement with the VNA and serves merely as a reference for the comparison with the estimated results. The comparison between the directly measured (gray) and the estimated results for the magnitude and the phase of the impedances are illustrated in Fig. 7. The results show a good correlation for the SPM (blue) and DPM (green) up to $200 \mathrm{MHz}$. The CDM (red) shows a better accuracy at higher frequency.

\section{Discussion}

The reason for the deviation between the directly measured impedance and the estimated results are mainly the measurement- and deembedding errors. The actual impedance at the end of wire cannot be measured explicitly, due to the existence of the fixtures. The measured impedances show an inductive behavior for the PCB with the low resistive component and a capacitive behavior for the PCB with the high resistive component. The reason for the inductive and capacitive behavior at the higher frequency range is the structure of the PCBs and the expected behavior of SMD resistors at higher frequencies. At the lower frequency range, the ability of the measurement instrument and the characteristics of the ferrite core inside the current probe are the main restrictions for performing the impedance measurement. However, at higher frequencies, the measurement results are very sensitive to the parasitic capacitive coupling between the conductive elements such as the probe's metallic frame, the probe's winding, the clamped conductor and the ground plane. In addition, the quality of the datasets obtained from the calibration setups to remove the probes' frequency response are significantly affected by the length of the clamped conductor and the added fixtures. Despite the expected inductive behavior of the current probes, the capacitive coupling is dominant and reduces the local characteristic impedance of the wire. Considering constant values for $Z_{0}$ and $\gamma$ for the entire length of the wire, including the section clamped by the probe and the area near the fixtures, reduces 

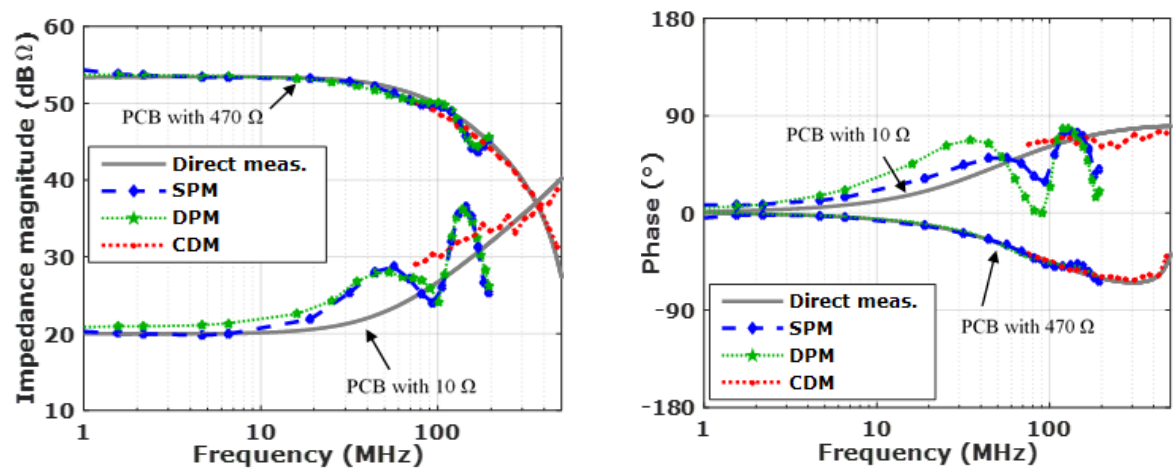

Figure 7. The Comparison between the directly measured (grey) and the estimated termination impedance for a high resistive PCB and a low resistive PCB with the SPM (blue), the DPM (green) and the CDM (red).

the accuracy of all methods. The high attenuation and the low dynamic of the current probes toward the loop impedance, due to the built-in resistive elements or the characteristics of the ferrite core limit the measurement bandwidth. Using current probes with smaller dimensions or assembling self-made current transformers with appropriate ferrite core characteristics can improve the measurement results. In order to improve the measurement results by suppressing the commonmode currents on the coaxial cables to the VNA, different ferrite beads appropriate for the desired frequency range are used during the entire measurement.

\section{Conclusions}

Based on the demand to improve BCI testing, comfortable and accurate termination impedance measurement methods are required. This paper presented three methods to estimate the impedances of the termination circuits in the immunity test setups using the clamp-on current probes. All of three methods were compared and experimentally validated for different resistive terminations. It is shown that the proposed methods are applicable without any need to disconnect the wire harness from the DUT. In particular, it has been highlighted that, the CDM estimates the impedance of both terminations with acceptable precision at the higher frequencies. However, the SPM and DPM can estimate the impedance of the termination up to $200 \mathrm{MHz}$. On the whole, the proposed methods are aimed to highlight the potential of the indirect measurement of termination impedances and extending the application of current probes for impedance measurement.

\section{Data availability}

Part of this research was done within cooperation projects and is subject to individual confidentiality agreements. Data used for this publication cannot be disclosed.
Edited by: F. Gronwald

Reviewed by: three anonymous referees

\section{References}

Adams, J. W., Cruz, J., and Melquist, D.: Comparison measurements of currents induced by radiation and injection, IEEE Transactions on Electromagnetic compatibility, 34, 360-362, 1992.

Pignari, S. and Canavero, F. G.: Theoretical assessment of bulk current injection versus radiation, IEEE Transactions on Electromagnetic compatibility, 38, 469-477, 1996.

Grassi, F., Spadacini, G., Marliani, F., and Pignari, S. A.: Use of Double Bulk Current Injection for Susceptibility Testing of Avionics, IEEE Transactions on Electromagnetic compatibility, 50, 524-535, 2008.

Hassanpour Razavi, S. A., Miropolsky, S., and Frei, S. Verbesserung der Korrelation zwischen dem BCI- und dem Antennenprüfverfahren für Kfz-Komponenten durch Anpassung von Verstärkerleistung und Position der BCI-Zange, EMVDüsseldorf, Germany, 2014

Junge, A.: Kontaktlose Verfahren zur breitbandigen Messung an Leitungen bei Hochfrequenz, Aachen, Shaker 2009.

Paul, C. R.: Analysis of Multiconductor Transmission Lines, John Wiley \& Sons, 1994.

Liu, G., Ding, Y., Chen, C., Kautz, R., Drewniak, J., Pommerenke, D., and Koledintseva, M.: A Dual-Current-Probe Method for Characterizing Common-Mode Loop Impedance, IEEE Instrumentation and Measurement Technology Conference, Colorado, USA, May 2003. 